\title{
OBIA ON COASTAL LANDFORM BASED ON STRUCTURE TENSOR
}

\author{
Sun Shuting, Liu Jianqiang and Zou Bin \\ Key laboratory of Space Ocean Remote Sensing and Application, \\ SOA, Beijing, China \\ National Satellite Ocean Application Service, Beijing, China \\ sst19910323@gmail.com \\ jqliu@mail.nsoas.org.cn \\ zoubin@mail.nsoas.org.cn
}

\begin{abstract}
This paper presents the OBIA method based on structure tensor to identify complex coastal landforms. That is, develop Hessian matrix by Gabor filtering and calculate multiscale structure tensor. Extract edge information of image from the trace of structure tensor and conduct watershed segment of the image. Then, develop texons and create texton histogram. Finally, obtain the final results by means of maximum likelihood classification with KL divergence as the similarity measurement. The study findings show that structure tensor could obtain multiscale and all-direction information with small data redundancy. Moreover, the method described in the current paper has high classification accuracy.
\end{abstract}

\section{KEYWORDS}

OBIA, Structure Tensor, Texton \& Coastal Zone

\section{INTRODUCTION}

Remote sensing with its large amount of information, access to information faster, shorter cycle, less restricted and so on provides a new method for the investigation of the coastal zone. In view of the characteristics of the coastal zone, the methods of land use classification are proposed. The original classification method of the coastal zone is based on pixel-based classification, such as Maximum Likelihood or ISODATA. These methods are suitable for low and medium spatial resolution remote sensing image; as for high-resolution images, information, such as texture information single pixel able to contain has its limitation. Thus, object oriented classification is widely used. That method firstly segment image to object, which is a series of homogeneous regions adjacent to each other. After that, classify these objects.

In recent years, some scholars have applied the object-oriented classification to the classification of coastal landform. In the year 2009, Wang Changying[1] has proposed a coastline extraction methods based on Apriori Algorithm, which can extract 5 different kind of coastline: artificial coastline, bedrock coastline, sandy coastline, muddy coastline and biological coastline. Using such method, the extraction accuracy can reach 3 pixels by using Landsat image $(30 \mathrm{~m})$.

Natarajan Meghanathan et al. (Eds) : ACITY, VLSI, AIAA, CNDC - 2016

pp. 69-80, 2016. (C) CS \& IT-CSCP 2016

DOI : $10.5121 /$ csit.2016.60907 
Proposed by Bigün[2] in 1987, structure tensor indicates the consistency of the matrix which was originally used for detection of linear symmetry. In recent years, structure tensor method is improved from 2 aspects:

In practice, the distance between objects and the viewpoint is different, in addition, objects have specific spatial scale. Therefore, single scale structure tensor is unable to describe texture information of different scales. Thus, scientists expand single scale structure tensor to multiscale structure tensor to enhance the capacity in describing image information, Sensitive to noise, structure tensor usually requires filtering. The most common filter is the Gaussian filter. Nonlinear structure tensor was proposed by M Rousson et al [3] in 2003, that is, Gaussian filter is replaced by nonlinear filter, which could preserve more information and restrain noise at the same time.

Multiscale structure tensor is able to extract multiscale and all-direction information with small data redundancy. In the past 30 years, it has been successfully applied in many fields such as image recovery [4], frequency and ridge estimation [5], texture analysis [6] and image processing in neural biology field [7]. In this paper, in order to develop multiscale structure tensor, Hessian matrix is constructed using Gabor wavelet and nonlinear diffusion filter is used to reduce the noise. Multiscale structure tensor serves as texture information about coastal areas.

It is difficult to directly compare the similarity of 2 objects due to different amount of pixels in different objects. In this paper, texton histogram is used to describe the information of the objects.

Texton is the fundamental microstructure of natural image. [8] A plurality of similar texture and spectral features in the same texture area can compose such microstructure. These measurement vectors have certain similarity. Typical vectors can be used to represent all similar vectors in the region and discard the others. Which can reduce amount of computation and to multivariable decoupling in order to reduce the correlation of vectors. The texton histogram is a typical application of this theory. In this method, New measurement vectors are obtained by clustering the old measurement vectors.

\section{STUDY AREA AND RESEARCH DATA}

The study area is located in the southeast of Hainan Island. As it's shown in figure 1.

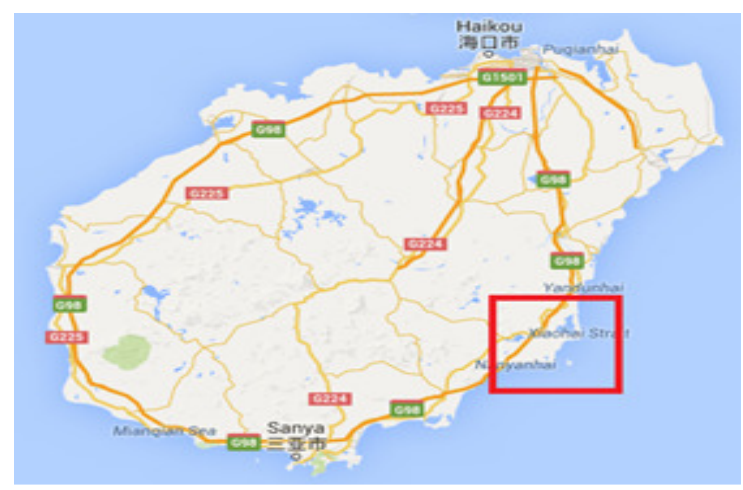

Figure 1. Location of Study Area 
The research data is the image of WorldView2, a high-resolution commercial remote sensing satellite launched by Globe Digital Company in October 2009. WorldView2 contains 8 multi spectral bands and 1 Panchromatic band. The Panchromatic resolution is $0.46 \mathrm{~m}$, and multi spectral resolution is $1.84 \mathrm{~m}$. In this paper, the image product has 4 bands (RGB and NIR) with resolution at $2 \mathrm{~m}$ (after image fusion).

\section{TEChNOLOGICAL PROCESS}

The technical process is shown in Figure 2.

First, the satellite image is filtered by Gabor to develop Hessian matrix, employ nonlinear diffusion filtering to develop structure tensor. Then the edge information of the image is obtained by analyzing the trace and conduct watershed segment. After that, the author utilizes the trace and eigenvalues of structure tensor, structure tensor and spectral information to develop textons and calculate texton histogram. Finally, the author carries out the maximum likelihood classification based on KL divergence to get the final classification result.

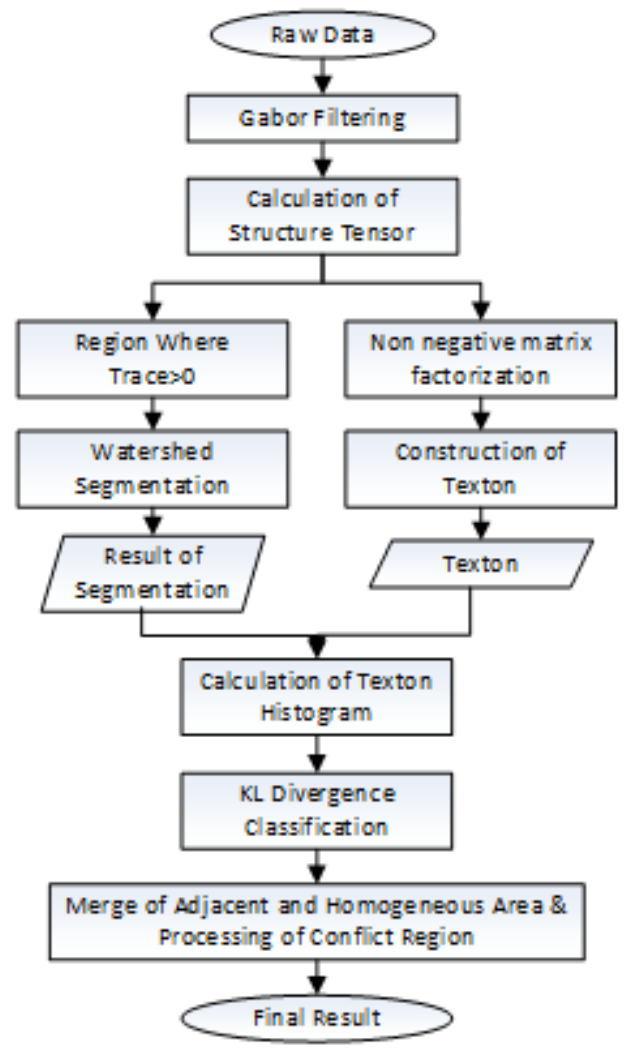

Figure 2. Technology Process

\section{STRUCTURE TENSOR}

For grey scale image I, the structure tensor is shown as Formula (4.1): 


$$
\mathrm{T}=k *\left|\begin{array}{ll}
I_{x}{ }^{2} & I_{x} I_{y} \\
I_{x} I_{y} & I_{y}{ }^{2}
\end{array}\right|
$$

Where $I_{x}$ is the partial derivative of the image in x direction and $I_{y}$ is the partial derivative in $\mathrm{y}$ direction. It is easy to extend Formula (4.1) to higher order as shown in Formula (4.2):

$$
\mathrm{T}=k *\left|\begin{array}{ll}
\sum\left(I_{x}{ }^{2}\right) & \sum\left(I_{x} I_{y}\right) \\
\sum\left(I_{x} I_{y}\right) & \sum\left(I_{y}{ }^{2}\right)
\end{array}\right|
$$

For multi spectral data, the structure tensor is the sum of the tensor product of each band.

Scheunders[9] has proposed the multiscale feature description method based on wavelet and the wavelet basis function $\gamma$ satisfies the Formula (4.3):

$$
\left\{\begin{array}{c}
\lim _{x, y \rightarrow \infty} \gamma(x, y)=0 \\
\int \gamma(x, y) d x d y=1
\end{array}\right.
$$

Where $\gamma$ is a kind of two dimensional differentiable function. Therefore, wavelet basis function can be used as multi scale convolution kernel. The elements in a Hessian matrix are obtained by convolving the image and calculating the partial derivative.

$$
\left(\begin{array}{c}
D_{x} \\
D_{y}
\end{array}\right)=a^{S}\left(\begin{array}{c}
\frac{\partial\left(I * \gamma_{S}\right)}{\partial x} \\
\frac{\partial\left(I * \gamma_{S}\right)}{\partial y}
\end{array}\right)
$$

Where a is the wavelet base (constant). $\gamma_{\mathrm{s}}$ is the wavelet basis function in scale s. Gabor wavelet is used as wavelet basis function based on the method of Shoudong Han et al [7].

\subsection{Gabor Filters}

Gabor filters is to divided signal into many small time intervals. Using Fourier transform to analyse each time intervals. The specific process is to add a Gaussian function to $f(t)$ as window function and then doing Fourier transform. As for the two-dimensional data, its window function is as follows [10]:

$$
g(x, y)=\frac{1}{2 \pi \sigma^{2}} e^{-\frac{1}{2}\left(\frac{x+y}{\sigma}\right)^{2}}
$$

Put it into Gabor transformation function:

$$
G_{f}=\int_{-\infty}^{\infty} f(t) g(t-b) e^{-i \omega t} d t
$$

Obtain Gabor basis function of two-dimensional discrete data:

$$
\mathrm{G}(x, y)=\frac{1}{2 \pi \sigma^{2}} e^{-\frac{1}{2}\left(\frac{x+y}{\sigma}\right)^{2}} \cos (2 \pi f(x \cos \theta+y \sin \theta))
$$

$\mathrm{f}$ is the frequency, determined by formula (5.4); $\theta$ is the angle, make it as $0^{\circ}, 45^{\circ}, 90^{\circ}, 135^{\circ}$ 


$$
\mathrm{f}=\frac{1}{2^{n} \cdot 2 \sqrt{2}}(n=0,1,2)
$$

Therefore, the calculation of the multiscale structure tensor is as Formula (4.9):

$$
\mathrm{T}=k *\left|\begin{array}{cc}
\sum\left(D_{x}^{2}\right) & \sum\left(D_{x} D_{y}\right) \\
\sum\left(D_{x} D_{y}\right) & \sum\left(D_{y}^{2}\right)
\end{array}\right|
$$

\subsection{Nonlinear Diffusion Filter}

PM diffusion is a nonlinear diffusion model proposed by Perona and Malik [11] in 1990. It is a kind of nonlinear adaptive PDE algorithm. The calculation process contains detection of edge and direction of the image. In non-edge area, the filter is isotropic of high smooth degree. In edge area, the filter is anisotropic with low smooth degree. The application of it to images could reduce noise and maintain or even enhance image characteristics at the same time.

Discrete nonlinear diffusion PM equation is shown in Formula (4.10):

$$
\partial \mathrm{T}(x, y)=\operatorname{div}\left(g\left(\sum_{i=1}^{2} \sum_{j=1}^{2}|\nabla T(i, j)|^{2}\right) \nabla T(x, y)\right)
$$

Where $T(x, y)$ is the structure tensor of pixel in the image with a coordinates of $(x, y), g$ is the edge detection function shown in Formula (4.11):

$$
g(|\nabla x|)=\frac{1}{|\nabla x|^{\varepsilon}+\delta}
$$

Where $\varepsilon$ is a positive constant to prevent the denominator as 0 ; and $\delta$ is the harmonic parameter of diffusion filter. This paper takes $\delta$ as 0.7 .

\section{IMAGE SEGMENTATION}

In this paper, watershed segmentation is applied, which is a region-based mathematical morphology segmentation method. It could obtain single pixel width, connection, enclosure and accurate outline.

Watershed algorithm can only segment single band image. In case of processing multi-bands image, certain algorithm should be used to reduce the dimension.

Structure tensor with higher order (4.2) can effectively process multi-band data. In addition, the Hessian matrix is developed by gradient, which contains the structure information of local neighbourhood areas without mutual offset of the gradients of rising edge and falling edge.

The trace of structure tensor of any pixel $(\mathrm{x}, \mathrm{y})$ of an image is calculated by Formula (5.1):

$$
\operatorname{trace}(x, y)=\operatorname{trace}\left(\begin{array}{cc}
T(x, y) & T(x, y+n) \\
T(x+m, y) & T(x+m, y+n)
\end{array} \mid\right)
$$

Where $m$ and $n$ is the length and width respectively of the image. In this paper, the part of trace $>0$ is selected as the gradient image. 


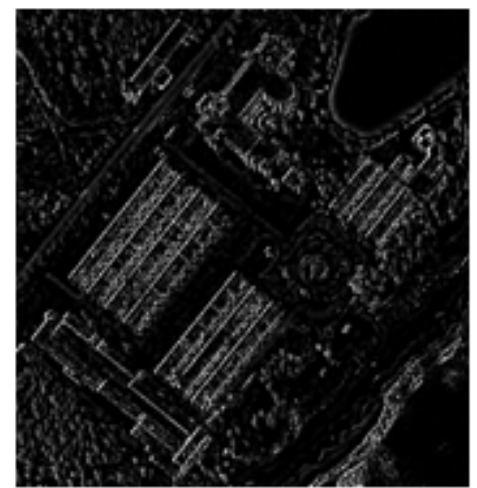

(a)

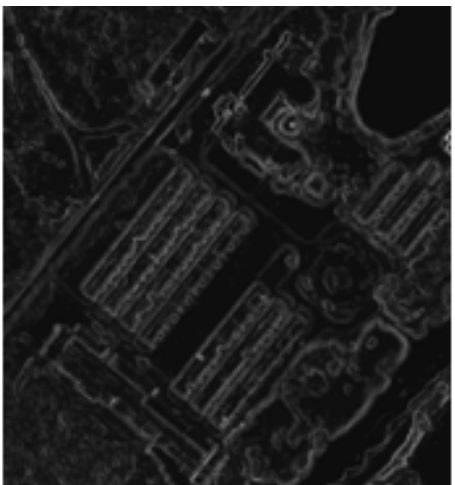

(b)

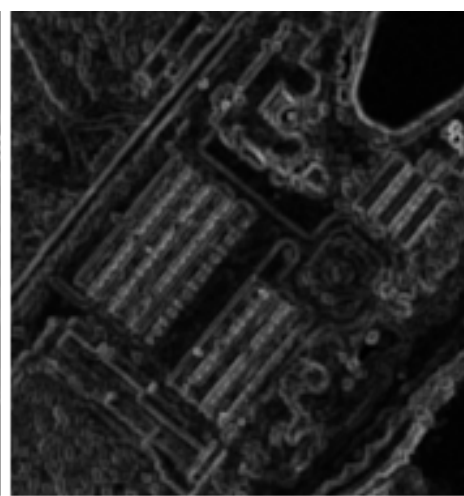

(c)

Figure 3. Comparison of the Results of CIE Transformation

In Figure 3, a is the gradient obtained by the method in this paper; $b$ is multidimensional gradient and $\mathrm{c}$ is morphological gradient. It can be seen that the trace of structure tensor can more effectively use the information of each band and obtain more accurate detection of the edge.

\section{CONSTRUCTION OF TEXTON}

OBIA requires describing objects with complex spectral and texture information and of different size after image segmentation. In this paper, the author develops texton to describe the information of object. Firstly, texture operator is selected; 4 bands spectral data is combined to develop textons. Then, the non-negative matrix factorization is employed to reduce the dimension.

\subsection{Texture Feature}

Texture operator selected is shown in Table 1. A total of 20 texture feature vectors are chosen as texture information of the object. Among them, the texture operator based on eigenvalues of the structure tensor is estimated by Formula (6.1)

$$
\mathrm{E}(x, y)=\frac{1}{1+B\left(\lambda_{1}-\lambda_{2}\right)^{2}}
$$

Where $\mathrm{B}$ is a constant, taking 1 in the current paper. $\lambda$ is the eigenvalue of structure tensor at the point $(\mathrm{x}, \mathrm{y})$.

Table 1. List of Texture Feature

\begin{tabular}{|l|l|l|}
\hline Texture Name & Dimension & Notification \\
\hline Structure Tensor & 12 & 3 scales, 4 directions a total of 12 dimensions \\
\hline Trace & 3 & As formula(5.1), 3 scales a total of 3 dimensions \\
\hline Eigenvalue & 3 & As formula(6.1), 3 scales a total of 3 dimensions \\
\hline NDWI & 1 & \\
\hline ARVI & 1 & \\
\hline
\end{tabular}

\subsection{Non negative matrix factorization}

In this paper, texture is reduced from 20 bands to 6-dimensions using non negative matrix factorization. Reducing computation load and reducing the correlation between bands. In Figure 
4, a shows the clustering results by using this method, b shows the result without using nnmf, c shows the clustering result by only using Spectral information.

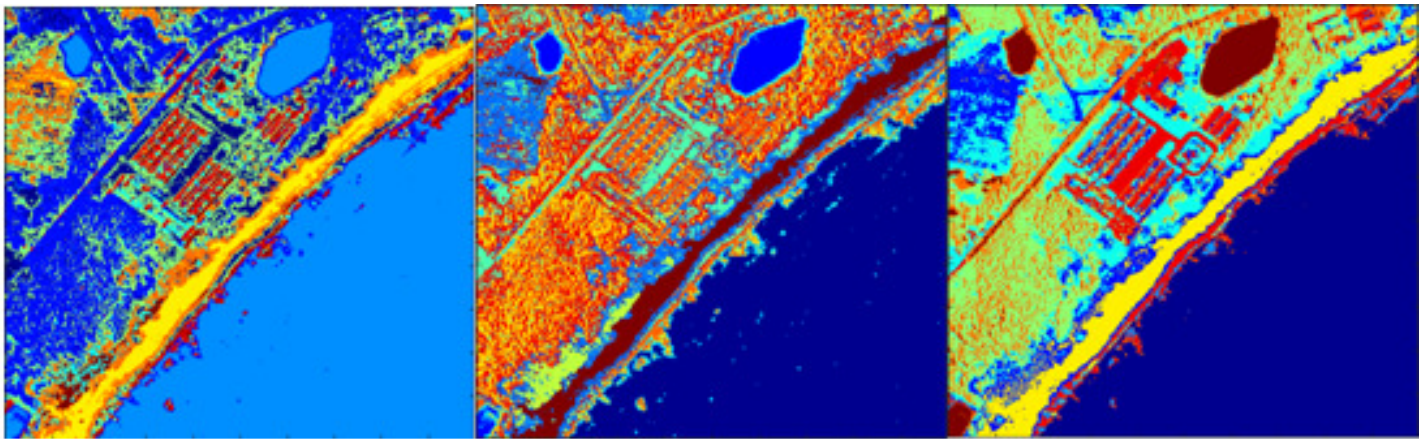

(a) (b) (c)

Figure 4. Results of Clustering of the Texton

The comparison of the results of the above three methods shows that texton can effectively identify most objects and is an effective method. NNMF not only reduces computation load, but also reduces the correlation between bands. It maintains more information without reducing classification accuracy after dimensionality reduction.

At a result, a 10 dimensional texton is obtained.

\section{Classification of The OBJeCtS}

The author divides the images into the following 7 categories: seawater, land water area, forest land, farm land, building, bare land and coastal (tidal zone) coastal (intertidal zone). based on characteristics of the image and Szuster B W's [12] method.

The vegetation is classified based on density degree in line with Ecker's standards [13] as well as the Ethnography of Hainan. Forest in the study area in the south-eastern end of Hainan Island is the forest land and other terrestrial vegetation is farm land. The coastal type is the muddy coast, which can be further divided into tidal and intertidal zones.

\subsection{Texton Histogram of the Training Sample}

With the calculation in Chapter 6, the author employs 10 dimension textons to describe the characteristics of the object. However, the author cannot directly compare two objects due to different amount of textons (i.e. pixels) in different objects. Therefore, Texton histogram is used in the current paper to describe the information of object so as to compare the similarity of two objects with different number of pixels. The process of generating texton histogram is as the followings:

a) Classify these textons into 9 groups by ISODATA and record the group of each texton.

b) Obtain texton histogram by count the frequency of the group of all textons in an object.

c) Turns different histogram in the same dimension by histogram normalization. 
Texton histogram of each class is shown in Table 2.

Table 2. Texton Histogram of the Training Sample

\begin{tabular}{|c|c|c|c|}
\hline Class & Texton Histogram & Class & Texton Histogram \\
\hline Seawater & $\begin{array}{ll} \\
\because \\
\because \\
\because\end{array}$ & $\begin{array}{l}\text { Land } \\
\text { Water } \\
\text { Area }\end{array}$ & 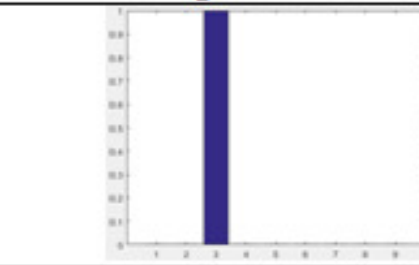 \\
\hline $\begin{array}{l}\text { Forest } \\
\text { Land }\end{array}$ & _ & Farmland & 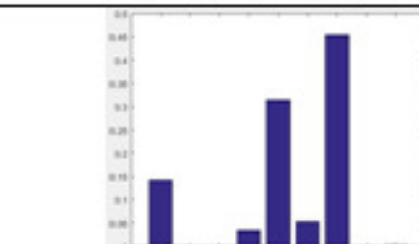 \\
\hline Buildings & 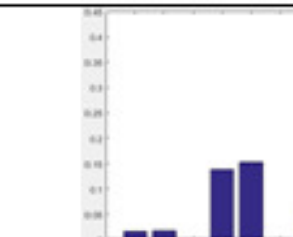 & Bare land & " \\
\hline Tidal & 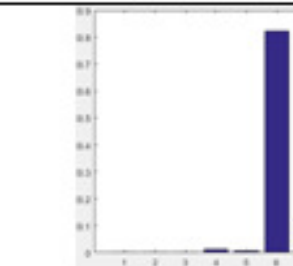 & Intertidal & 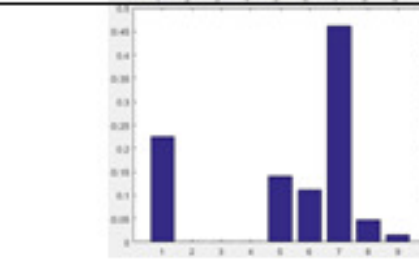 \\
\hline
\end{tabular}

\subsection{Maximum Likelihood Classification}

The maximum likelihood is used in the current paper to classify the objects. Texton histogram reflects the distribution of probability of various kinds of textons because it is the probability statistics of texton types contained in the object. Therefore, this paper employs KL divergence as similarity measurement as shown in the following Formula:

$$
D(p \| q)=\sum p(x) \log \frac{p(x)}{q(x)+a}
$$

Where $\mathrm{p}$ and $\mathrm{q}$ are the probability distribution of $\mathrm{x}$. In this paper, $\mathrm{p}$ is the texton histogram of subject sample and $\mathrm{q}$ is the texton histogram of control sample. To avoid denominator is 0 , constant a is added. This paper takes 0.01 as a value. The classification of an object can be identified until the KL divergence is the minimum among them and it's smaller than the threshold value $\mathrm{t}$; and $\mathrm{t}$ is considered as 0.1 . 


\subsection{Processing of Classification Result}

Watershed algorithm cannot avoid the over-segmentation, so the phenomenon exists that 2 areas adjacent to each other are of the same class The border width of Watershed algorithm is fixed as 1. Kang, T. Yung's [14] Bridge algorithm can eliminate such boundary. Bridge algorithm is defined as a connection of connected points. In the binary image, for a point whose value is 0 , if there is more than 2 points with value of 1 as it neighbourhood, value of the point is 1 . As it's shown in Formula (7.2)

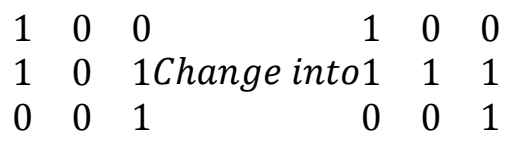

Texton histogram of seawater and land water area is similar, water areas are combined after extraction, mainly, the largest area is seawater.

\section{RESULT OF THE EXPERIMENT AND ASSESSMENT OF THE ACCURACY}

The study area is shown in Figure 6. The image is taken by Worldview2 on June $27^{\text {th }}, 2011$, located at $18^{\circ} 46^{\prime} 39^{\prime \prime} \mathrm{N}, 110^{\circ} 29^{\prime} 45^{\prime \prime} \mathrm{E}$ with the size at $1000 \times 1000$ pixels.

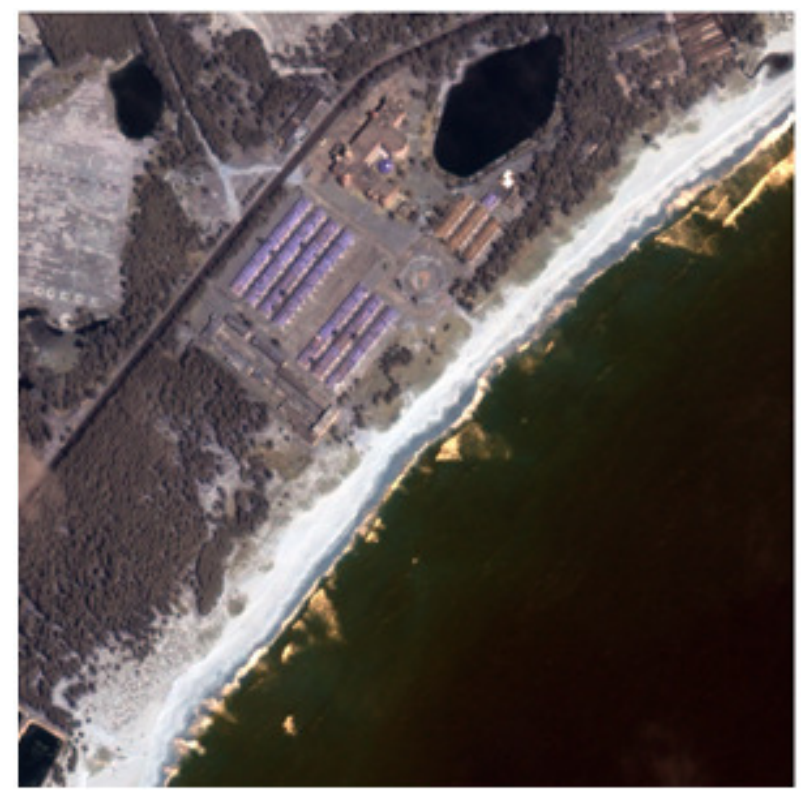

Figure 5 Original Image of the Study Area 


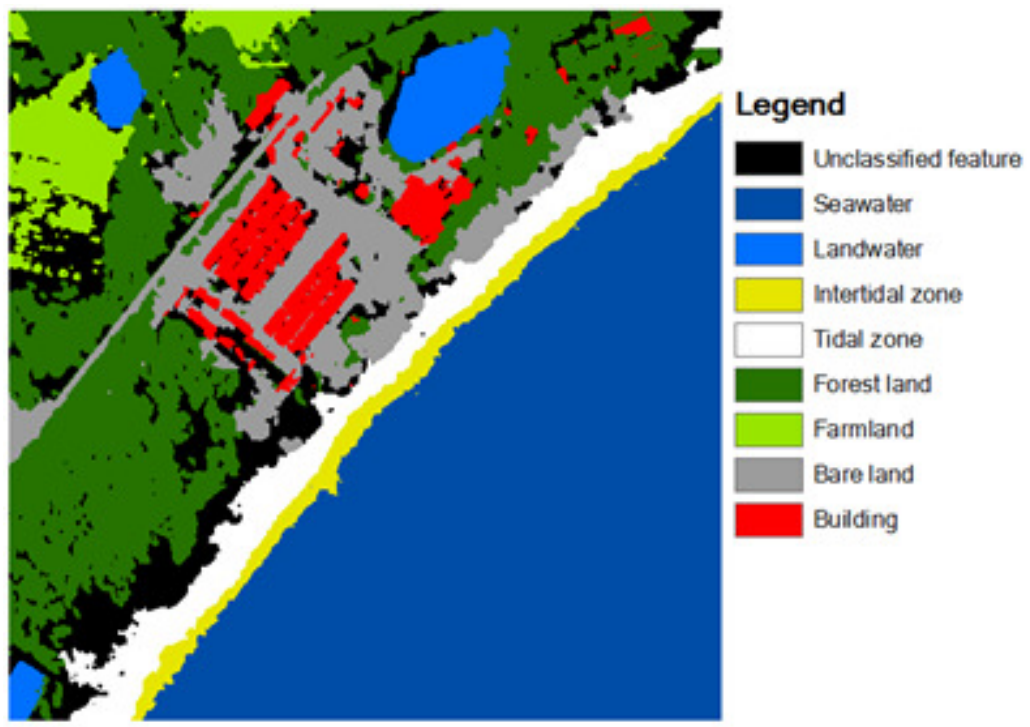

Figure 6. the Result of Classification

Table 3. Confusion Matrix

\begin{tabular}{|l|l|l|l|l|l|l|}
\hline \multirow{2}{*}{ Landform } & \multicolumn{2}{|l|}{ Method in this Paper } & \multicolumn{2}{l|}{$\begin{array}{l}\text { Maximum } \\
\text { Likelihood }\end{array}$} & \multicolumn{2}{l|}{ Method Without NIR } \\
\cline { 2 - 7 } & $\boldsymbol{P A}(\boldsymbol{\%})$ & $\boldsymbol{U A}(\boldsymbol{\%})$ & $\boldsymbol{P A}(\boldsymbol{\%})$ & $\boldsymbol{U A ( \% )}$ & $\boldsymbol{P A}(\boldsymbol{\%})$ & $\boldsymbol{U A ( \% )}$ \\
\hline Seawater & 100 & 100 & 90.5 & 95 & 90.9 & 100 \\
\hline Land water & 100 & 100 & 100 & 95 & 100 & 95 \\
\hline Forest Land & 90.0 & 90 & 80.0 & 80 & 81.8 & 90 \\
\hline Farm Land & 84.2 & 80 & 73.7 & 70 & 75 & 75 \\
\hline Bare Land & 73.9 & 85 & 62.5 & 75 & 65 & 65 \\
\hline Building & 90.0 & 90 & 85.7 & 90 & 80 & 80 \\
\hline Tidal & 89.5 & 85 & 76.2 & 80 & 77.3 & 85 \\
\hline Intertidal & 94.7 & 90 & 93.3 & 70 & 88.2 & 75 \\
\hline OA(\%) & 90.0 & 81.9 & & 83.1 & \\
\hline Kappa & 0.886 & 0.793 & 0.807 & \\
\hline
\end{tabular}

In table 3, first column shows the result of method in this paper, second column is the result using Maximum Likelihood on pixel. Third column shows the result without NIR data using structure tensor method (without NDWI and ARVI texture). Training sample of all methods are of the same. It is found by comparison of the results of the above three methods:

Firstly, overall accuracy of Maximum Likelihood is higher than $80 \%$, similar to structure tensor method without NIR data. With NIR information, quality of the texture has been significantly improved. Besides, all methods have a high accuracy among landform with obvious characteristics like seawater which represent spectral information of the image is fundamental.

Secondly, method of this paper have an obvious improvement on classification of bare land and farmland and that of method without NIR data is passable. Therefore, structure tensor is an effective texture algorithm on representation of image local information. 
Thirdly, overall accuracy of the method described in this paper can reaches $90.0 \%, 8.9 \%$ higher than that of the maximum likelihood method with 0.093 rise of Kappa coefficient. In conclusion, method of this paper has high classification accuracy.

\section{CONCLUSiOnS}

The findings of the current study demonstrate that structure tensor is an excellent texture operator because of its capacity in obtaining all-direction and multiscale information with small data redundancy. The overall accuracy of the OBIA method based on structure tensor adopted in this paper is above $90 \%$ and this method can be effectively applied in classifying coastal landforms.

Meanwhile, the author finds that there is quite big room for improvement of the current method as structure tensor texture can be further optimized by energy function of wavelet.

\section{FUNDING}

Project supported by Welfare Special Industry Fund of Marine Public Welfare, SOA of China (Grant No. 201505014).

\section{REFERENCES}

[1] 王常颖. 基于数据挖掘的遥感影像海岸带地物分类方法研究 [D]. 青岛: 中国海洋大学, 2009 .

[2] Bigün J, Granlund G H, Wiklund J. Multidimensional orientation estimation with applications to texture analysis and optical flow[J]. IEEE Transactions on Pattern Analysis \& Machine Intelligence, 1991 (8): 775-790.

[3] Rousson M, Brox T, Deriche R. Active unsupervised texture segmentation on a diffusion based feature space[C]//Computer vision and pattern recognition, 2003. Proceedings. 2003 IEEE computer society conference on. IEEE, 2003, 2: II-699-704 vol. 2.

[4] Chierchia G, Pustelnik N, Pesquet-Popescu B, et al. A nonlocal structure tensor-based approach for multicomponent image recovery problems[J]. Image Processing, IEEE Transactions on, 2014, 23(12): 5531-5544.

[5] Mikaelyan A, Bigun J. Frequency and ridge estimation using structure tensor[C]//Biometric Technologies in Forensic Science (BTFS 2013), Nijmegen, Netherlands, October 14-15, 2013. Radboud University Nijmegen, 2013: 58-59.

[6] Han S, Wang X. Texture Segmentation Using Graph Cuts in Spectral Decomposition Based Riemannian Multi-Scale Nonlinear Structure Tensor Space[J]. International Journal of Computer Theory and Engineering, 2015, 7(4): 259.

[7] Budde M D, Frank J A. Examining brain microstructure using structure tensor analysis of histological sections[J]. Neuroimage, 2012, 63(1): 1-10.

[8] Tuceryan M, Jain A K. Texture analysis[J]. The handbook of pattern recognition and computer vision, 1998, 2: 207-248.

[9] Scheunders P. A multivalued image wavelet representation based on multiscale fundamental forms.[J]. IEEE Transactions on Image Processing, 2002, 11(5):568-575. 
[10] Lee T S. Image representation using 2D Gabor wavelets[J]. Pattern Analysis and Machine Intelligence, IEEE Transactions on, 1996, 18(10): 959-971.

[11] Lindeberg T. Scale-space theory in computer vision[M]. Springer Science \& Business Media, 2013.

[12] Szuster B W, Chen Q, Borger M. A comparison of classification techniques to support land cover and land use analysis in tropical coastal zones[J]. Applied Geography, 2011, 31(2): 525-532.

[13] Eckert S. Improved Forest Biomass and Carbon Estimations Using Texture Measures from WorldView-2 Satellite Data[J]. Remote Sensing, 2012, 4(4):810-829.

[14] Kong T Y, Rosenfeld A. Digital topology: introduction and survey[J]. Computer Vision, Graphics, and Image Processing, 1989, 48(3): 357-393.

\section{AUTHORS}

Sun Shuting (1991.3 )

Master student of National Satellite Ocean Application Service, China.

Research direction: OBIA and Antarctic Sea Ice.

\section{Liu Jianqiang (1964.9 )}

Research Fellow,

Deputy director of National Satellite Ocean Application Service, China.

Research direction: Construction of Ocean Satellite and Ocean Remote Sensing
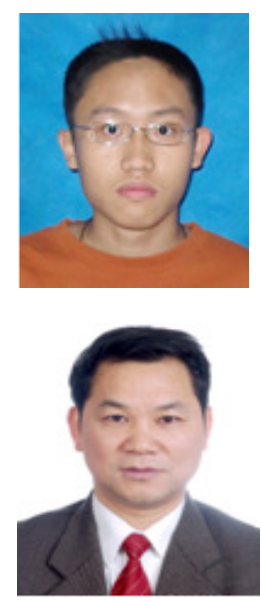

\section{Zou Bin (1969.2 )}

Research Fellow of National Satellite Ocean Application Service, China.

Research direction: Ocean Remote Sensing

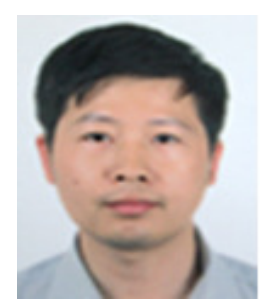

